\title{
Urban Land Cover Classification using WorldView-2 Images and C4.5 Algorithm
}

\author{
Bárbara Maria Giaccom Ribeiro \\ DPI - Image Processing Division \\ INPE - National Institute for Space Research \\ São José dos Campos - SP, Brazil \\ bgiaccom@gmail.com
}

\author{
Leila Maria Garcia Fonseca \\ DPI - Image Processing Division \\ INPE - National Institute for Space Research \\ São José dos Campos - SP, Brazil \\ leila@dpi.inpe.br
}

\begin{abstract}
Mapping of urban land cover using remote sensing technology has been widely explored, especially with the recent availability of high resolution images and object-based analysis techniques. This study uses the InterIMAGE software and WorldView-2 sensor imagery, two recent technologies useful for urban studies, to classify land cover in a metropolitan area of São Paulo, Brazil. Therefore, this work aims to compare the classification performance of two urban land cover thematic maps produced by the object-based image analysis (OBIA) and C4.5 data mining algorithm. The results showed that data mining technique presented classification performance similar to the OBIA method but in a reduced computational time once the entire processing is optimized.
\end{abstract}

\section{INTRODUCTION}

Urban areas are dynamic systems of great complexity, materializing the results of human action over the natural environment. In these areas, where most human activities are developed, significant changes of natural resources occur and impact on the ecosystems at many different scales.

The use of up-to-date and detailed information about urban land cover is strategic for urban planning and management in present times, involving issues related to the recent massive urban sprawl and densification, climate change, and the need for environmental protection. Urban land cover mapping using remote sensing technology has been widely explored, especially with the recent availability of high-resolution images [1]. The information extracted from these products can be used to develop medium and long-term investments planning, monitor the increasing demands for infrastructure and social equipment, and support the elaboration of public policies in compliance with environmental guidelines and targeted to provide better quality of life to society.

Aerophotogrammetric survey is one of the oldest and most traditional sources for the generation of land cover maps. Widely explored, and used especially for developing urban studies, this is a costly procedure, and it is still usually executed only on demand. On the other hand, remote sensing as a source of information provides a synoptic view, systematic acquisition, and comparatively lower costs.

The use of remote sensing imagery in urban areas is growing at a rapid pace as the potential of remote sensing is demonstrated and confirmed in many different applications. However, a prerequisite for extracting information from these products is the knowledge of the spectral behavior of surface objects to be analyzed and the factors that influence their behavior. This became possible due to the fact the sensors aboard new satellites are increasingly suited to urban studies, due to the enhancement in spatial, spectral and radiometric resolutions.

Urban studies concerning land cover mapping were first developed using Landsat TM images at a regional scale or addressing to urban expansion and dispersion. In 1999, the IKONOS satellite was successful launched, and its data provided more accurate analysis of urban environment. Thereat, in 2001, QuickBird-2 brought an increase in spatial resolution, bringing data to sub-meter level. Then, in 2008, GeoEye-1 started providing less than 0.5 meter images ${ }^{\mathbf{A}}$. These sensors enabled the development of an enormous amount of urban studies. Nevertheless, besides the enhancement of spatial resolution (PAN images: $1 \mathrm{~m}, 0.61 \mathrm{~m}$ and $0.41 \mathrm{~m}$, respectively), the spectral resolution remained similar, with no improvements on the standard "blue, green, red, near infrared" bands. In October 2009, the launch of WorldView-2 sensor opened new perspectives for studying spectral properties of urban targets. This is the first high spatial resolution orbital sensor $\left(0.46 \mathrm{~m}^{\mathrm{A}}\right.$ in panchromatic band and $1.84 \mathrm{~m}^{\mathbf{A}}$ in multispectral bands) with eight multispectral bands ranging from Blue to Near Infra-Red parts of the electromagnetic spectrum. In comparison to other orbital sensors with sub-meter resolution, the availability of four more bands in the WorldView-2 sensor has significantly increased its potential for urban land cover mapping, increasing accuracy from $65-70 \%$ to $85-90 \%[2,3,4,5]$.

When using automated methods to map the urban environment, a greater range of spectral bands is essential to discriminate the very similar land cover material such as fiber cement tiles (with and without asbestos), pigmented concrete tiles, flat-slabs roofs, and concrete paving [6]. On the other hand, the high-spatial resolution images acquired by the available sensors grant the human interpreter the ability to perform very accurate manual classification. This configures a very timeconsuming task, as well as building semantic networks to classify images based on human knowledge. Software that performs Object-Based Image Analysis (OBIA) provides a great number of object attributes and different ways to model the semantic network, which make the task of classification lengthy and complex. Determining the most relevant features to be used in classification routines is not always an easy task when

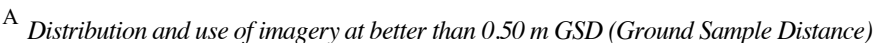
$P A N$ and $2.0 \mathrm{~m}$ GSD multispectral is subject to prior approval by the U.S. Government.
} 
conventional exploratory analyses are carried out (e.g., scatter plot, histograms, feature values shown in grey levels, etc.).

Data mining tools can increase the potential for the analysis of remote sensing data [7] once these techniques quickly select the most representative attributes for each class and generate simple classification rules. Thus, attribute selection methods became very attractive for remote sensing studies concerning urban areas due to the hundreds of spectral, texture and geometric attributes that can be used in OBIA classification routines. Despite the wide exploration of urban land cover classification based on high resolution imagery, few studies have applied automatic methodologies to define parameters and features for object-based image classification.

This study compares two urban land cover thematic maps produced by "manual" OBIA and data mining techniques. The experiments were developed in the InterIMAGE system, which is under development by PUC-RJ (Pontifical Catholic University - Rio de Janeiro) and INPE (Brazilian National Institute for Space Research), briefly described in the next section. The classification accuracy for both thematic maps was assessed through the confusion matrix, Kappa and conditional-Kappa indices.

\section{A. InterIMAGE System}

InterIMAGE is an open source and free access framework for knowledge-based image classification that provides high capacity for customization and extension tools. According to the developers [8,9], it is a multiplatform framework implemented for LINUX and Windows operational systems. The System is coded in $\mathrm{C}++$ and uses the QT4 crossplatform application development framework [10], the Visualization Toolkit (VTK) class library [11], and Terralib [12], which is a GIS classes and functions library developed at INPE. InterIMAGE provides support for the integration of image processing operators in the interpretation process and, as such, operators are treated as external programs by its control mechanism; they can be coded in any computer language and can even be proprietary programs [8].

InterIMAGE's interpretation process is guided by a knowledge model, which embodies and explicitly represents prior knowledge about a particular geographic region and about the objects expected to be found in it. The knowledge model can correspond to the user's prior knowledge or the knowledge acquired by analyzing the input data (RS images or GIS layers) through the tools provided by the system [8]. The classification strategy is based on a knowledge model structured as a semantic network defined by the user. The classification process has two steps: the Top-Down and the Bottom-Up [9].

In the Top-Down step, the system descends the semantic network, triggering the so-called holistic operators. Holistic operators are image processing operators, external to the system's core and specialized in the detection of a certain class. For the detection of objects from the corresponding class, holistic operators usually perform three procedures: segmentation (or import GIS data), attribute extraction, and classification. The geographic regions detected by a holistic operator inserted into a given node are transmitted as masks ("hypothesis") to its child nodes on the lower level of the semantic network, where its own holistic operators will work.
In the Bottom-Up step, the system ascends the semantic network, solving spatial conflicts between hypotheses based on user-defined rules inserted in every node that is not a leaf node. Doing so, the system either partially or totally discards the hypotheses or turns them into instances (i.e., validates the hypotheses). The user-defined rules may involve additional logical selections. If spatial conflicts still remain after the hypotheses rejection by these additional logical selections, they are solved either by the supervised definition of priority for the classes or by the competition of membership values given by user-defined fuzzy membership functions.

The final instance network results from the Bottom-Up analysis: when bottom-up operators reach the top of the semantic network, labeled images are created for each different level of the semantic network.

\section{B. C4.5 Decision Tree Algorithm}

The InterIMAGE framework offers a suit of basic operators assembled with the classes and functions supplied by the TerraLib library [12].

The C4.5 decision tree algorithm works in the following way: each node of the decision tree matches an attribute and each arc matches a value range of that attribute. The expected attribute value is defined by the path from the root to each leaf. The most representative attribute is associated with each node. The entropy is calculated to assess how informative a node is. The larger the entropy, the more information is necessary to characterize the data. The goal is to associate with a node the attribute that minimizes the data entropy [13].

After associating the attribute with a node, the decision tree algorithm defines a threshold value for each arc. The threshold is computed by nearest neighbor algorithm. Firstly, the algorithm calculates the Euclidian Distance from the training samples to an instance of the data. The instance will be assigned to the class that is closest to it in the space of attributes [14]. The C4.5 algorithm removes unnecessary nodes through the pruning procedure, producing the shortest tree possible [14]. The number of instances in each leaf also controls the size of the tree. The lower the number of instances, the more precise the classification for the training set is.

When using this tool, the user is responsible for defining the best parameters to segment the image and for selecting samples for each class. The processing that follows is developed within the InterIMAGE system, which generates the final classification and the decision tree (as a separate text file).

\section{METHODOLOGY}

The methodological steps are summarized in the block diagram in Fig. 1.

\section{A. Study Area}

The study area is located along the western section of the Rodoanel Mário Covas, a ring-road that surrounds the metropolitan area of São Paulo, Brazil. The chosen area addresses a wide range of urban targets, such as shrubs and trees, different types of roofs, buildings with variable heights, warehouses, etc.. These urban objects are frequently spatially arranged in a dense and complex manner and can be found in different states of conservation. 
Moreover, despite having similar colorations, these objects have very different chemical compositions and physical properties.

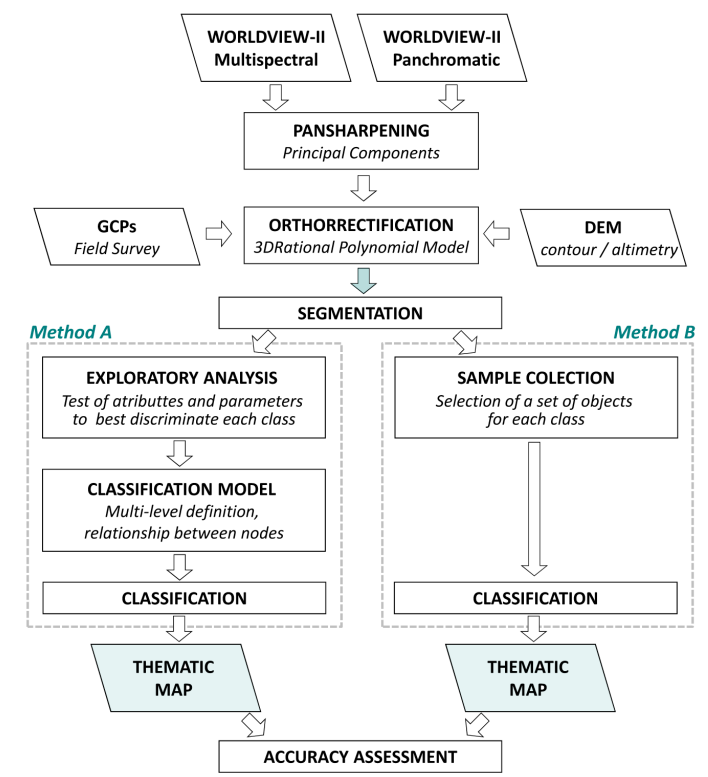

Figure 1. Methodological procedures for urban land cover mapping.

\section{B. Pre-processing}

A pan-sharpening technique based on Principal Components was applied to process the WorldView-2 multispectral and panchromatic bands to produce a hybrid image with 8 spectral bands and spatial resolution equal to $0.5 \mathrm{~m}$.

After fusion processing, the image was orthorrectified by applying the 3D rational polynomial method. The values of RMSE (Root Mean Square Error) obtained were $1.15 \mathrm{~m}$ and $1.07 \mathrm{~m}$, for the $\mathrm{E}$ and $\mathrm{N}$ planimetric components, respectively.

\section{Segmentation}

The segmentation was performed by InterIMAGE v1.33 [15], using TerraAIDA Baatz\&Schäpe algorithm [16]. The same segmentation parameters were used for both experiments.

\section{Land Cover Classification Analysis}

Ten land cover classes were established considering the possibility of their identification in the study area: GRASS AND Shrubs, Trees, Bare Soil, Clay Tile Roof, Metallic Roof, PVC Roof (Polyvinyl Chloride), Asphalt, Clear and DARK CEMENT MATERIAL, and SHADOW.

At this point, the methodology was divided into two parts: in the so called Method A, the behavior of each land cover class was assessed by the user through feature exploratory analysis. The semantic network was manually built. In the top-down step, hypothesis clusters were generated for each land cover class. The classification was carried out in the bottom-up steps, solving conflicts among the classes. Conflicts were solved by giving priority to classes whose attributes would distinguish them better with fewer omission and commission errors.

The Method $B$ was performed using machine learning and data mining techniques. After selecting 15 samples for each land cover class, the "TA_C45_Classifier" was settled as a top- down operator. The algorithm used the collected samples and a set of possible attributes (pre-selected by the user) to classify each remaining object into one of the possible classes. By using this approach, no conflicts between classes are generated, and the user has no further interaction on deciding the result.

The same machine was used in both procedures, which main characteristics are: processor: Intel Core 2 Duo $(2.53 \mathrm{GHz}, 3$ MB L2 cache); memory (RAM): 8GB DDR2, 800MHz; hard disk: SATA, 500GB (5400RPM); video card: onboard Intel Graphics Media Accelerator 4500MHD; and 64 bits operational system. The classification procedures consumed 168 minutes through Method A, and 35 hours through Method B.

\section{E. Classification Accuracy Evaluation}

The classification was assessed through the confusion matrix, Kappa and conditional-Kappa indices. From the confusion matrix, two descriptive measures were calculated: the user overall accuracy and the producer overall accuracy $[16,17,18]$.

\section{RESULTS}

Classifying the Worldview-2 image through Methods $A$ and $B$, as illustrated in Fig. 2, produced the thematic maps.

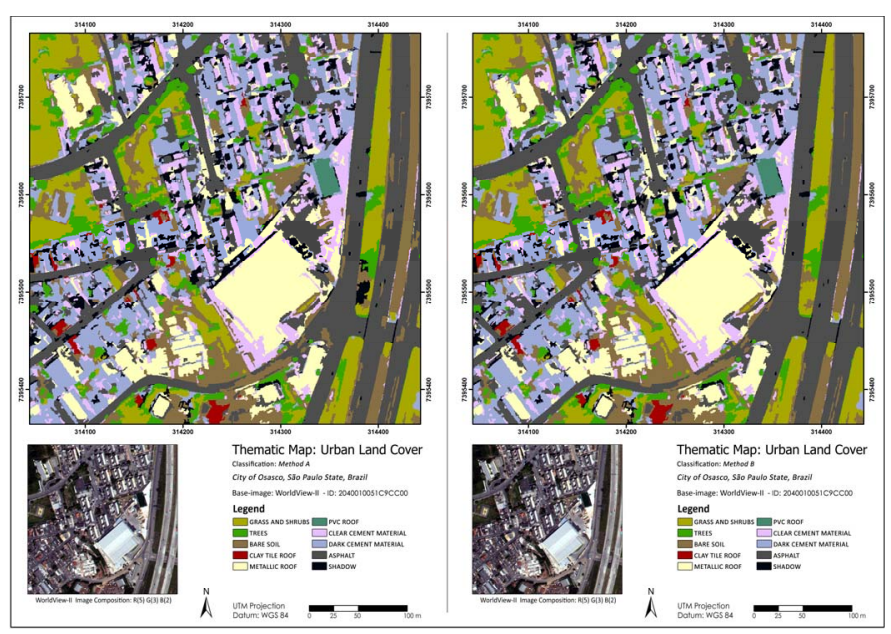

Figure 2. Thematic maps obtained from Method A (left) and Method B (right).

Statistical analysis indicated that the thematic map produced through Method $B$ is slightly - but not significantly - different from the one obtained by Method A. The overall accuracy and Kappa values for the Method B thematic map were a little higher than that obtained from Method A.

TABLE I. OVERALL ACCURACY, KAPPA INDEX AND VARIANCE OF THE KAPPA INDEX FOR THE CLASSIFICATIONS.

\begin{tabular}{|c|c|c|c|}
\hline $\begin{array}{c}\text { Thematic } \\
\text { Map }\end{array}$ & Kappa Index & $\begin{array}{c}\text { Overall } \\
\text { Accuracy }\end{array}$ & $\begin{array}{c}\text { Kappa } \\
\text { Variance }\end{array}$ \\
\hline Method A & 0.8134 & 0.8387 & 0.000644 \\
\hline Method B & 0.8340 & 0.8566 & 0.00060 \\
\hline
\end{tabular}

Overall, the discrimination between all classes was a little better achieved for Method B than for Method A, which was evaluated through the conditional-Kappa index and the user and producer accuracies. TABLE II shows the confusion matrices for both classifications, comparing each classified result with ground truth. User and producer accuracies are also related. 
TABLE II. CONFUSION MATRICES OF METHOD A AND METHOD B CLASSIFICATIONS.

\begin{tabular}{|c|c|c|c|c|c|c|c|c|c|c|c|c|}
\hline $\begin{array}{c}\text { Method } \\
A\end{array}$ & 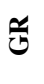 & 刍 & $\mathscr{\infty}$ & 苯 & 兰 & $\sum_{U}$ & $\sum^{\sum}$ & के & $\underset{\varpi}{\varpi}$ & $\sum_{2}^{u}$ & 氙 & \் \\
\hline GR & 24 & 5 & 0 & 0 & 0 & 0 & 0 & 0 & 0 & 0 & 29 & 0,828 \\
\hline TR & 0 & 12 & 1 & 0 & 0 & 0 & 0 & 0 & 3 & 0 & 16 & 0,750 \\
\hline BS & 3 & 0 & 22 & 1 & 0 & 0 & 2 & 2 & 1 & 0 & 31 & 0,710 \\
\hline CTR & 0 & 0 & 0 & 10 & 0 & 0 & 0 & 0 & 0 & 0 & 10 & 1 \\
\hline MR & 0 & 0 & 0 & 0 & 24 & 4 & 0 & 1 & 0 & 0 & 29 & 0,828 \\
\hline CCM & 0 & 0 & 0 & 0 & 2 & 23 & 2 & 1 & 0 & 0 & 28 & 0,821 \\
\hline DCM & 0 & 1 & 0 & 0 & 1 & 1 & 39 & 0 & 0 & 1 & 43 & 0,907 \\
\hline ASP & 0 & 0 & 0 & 0 & 1 & 2 & 7 & 60 & 0 & 0 & 70 & 0,857 \\
\hline SHA & 1 & 0 & 1 & 0 & 0 & 0 & 1 & 0 & 10 & 0 & 13 & 0,769 \\
\hline PVC & 0 & 0 & 0 & 0 & 0 & 0 & 0 & 0 & 0 & 10 & 10 & 1 \\
\hline TOTAL & 28 & 18 & 24 & 11 & 28 & 30 & 51 & 64 & 14 & 11 & 279 & \\
\hline
\end{tabular}

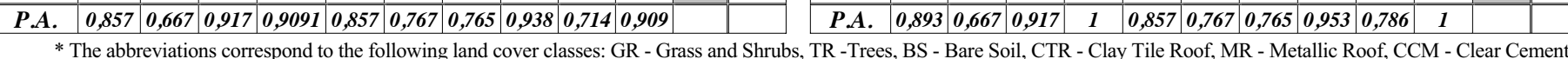

\begin{tabular}{|c|c|c|c|c|c|c|c|c|c|c|c|c|}
\hline $\begin{array}{c}\text { Method } \\
\text { B }\end{array}$ & 嵌 & 뜰 & $\mathscr{\theta}$ & 艺 & 米 & $\sum_{U}$ & $\sum_{\bigcup}$ & के & $\overleftrightarrow{\vec{\sigma}}$ & $e_{2}^{2}$ & 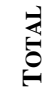 & 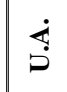 \\
\hline GR & 25 & 5 & 0 & 0 & 0 & 0 & 0 & 0 & 0 & 0 & 30 & 0,833 \\
\hline TR & 0 & 12 & 1 & 0 & 0 & 0 & 0 & 0 & 2 & 0 & 15 & 0,800 \\
\hline BS & 3 & 0 & 22 & 0 & 0 & 0 & 2 & 1 & & 0 & 28 & 0,786 \\
\hline CTR & 0 & 0 & 0 & 11 & 0 & 0 & 0 & 0 & 0 & 0 & 11 & 1 \\
\hline MR & 0 & 0 & 0 & 0 & 24 & 4 & 0 & 1 & 0 & 0 & 29 & 0,827 \\
\hline CCM & 0 & 0 & 0 & 0 & 2 & 23 & 2 & 1 & 0 & 0 & 28 & 0,821 \\
\hline DCM & 0 & 1 & 0 & 0 & 1 & 1 & 39 & 0 & 0 & 0 & 42 & 0,929 \\
\hline ASP & 0 & 0 & 0 & 0 & 1 & 2 & 7 & 61 & 1 & 0 & 72 & 0,847 \\
\hline SHA & 0 & 0 & 1 & 0 & 0 & 0 & 1 & 0 & 11 & 0 & 13 & 0,846 \\
\hline PVC & 0 & 0 & 0 & 0 & 0 & 0 & 0 & 0 & 0 & 11 & 11 & 1 \\
\hline \begin{tabular}{|l|} 
TOTAL \\
\end{tabular} & 28 & 18 & 24 & 11 & 28 & 30 & 51 & 64 & 14 & 11 & 279 & \\
\hline
\end{tabular}
Material, DCM - Dark Cement Material, ASP - Asphalt, SHA - Shadow, PVC - PVC Roof (Polyvinyl Chloride); P.A. stands for Producer Accuracy and U.A., for User Accuracy.

\section{CONCLUSION}

Using two technologies that are new and still little explored in urban studies, this work aimed to explore the WorldView-2 sensor imagery and the free open-source software InterIMAGE [15] for urban land cover classification, building a quantitative comparison between two classification methods. We also evaluated how the $\mathrm{C} 4.5$ algorithm could save time spent on semantic network construction and attribute selection tasks.

Although the manually built model is suitable to receive as many adaptations as necessary to perform a satisfactory classification, the user may also obtain good results by applying data mining techniques in OBIA. The use of the $\mathrm{C} 4.5$ algorithm in the classification process proved to enhance the classification, producing classification performance similar to OBIA methods, with sensitive time saving. In this case, the interpreter is only responsible for choosing a representative set of samples for each land cover class, which will be used by the $\mathrm{C} 4.5$ algorithm to perform the image analysis.

The C4.5 algorithm, integrated with other image analysis tools, presented great potential in high-resolution image analysis for urban applications. Finally, this work reinforces the gain on time and performance provided by the use of machine learning algorithms and data mining techniques in the classification process, which increased the speed and also reduced the empirical nature of attribute selection and network construction.

\section{ACKNOWLEDGMENT}

The authors would like to acknowledge CNPq (National Counsil of Technological and Scientific Development) for financial support during the realization of this study; Digital Globe, represented by Ph.D. Kumar Navulur, who supplied the WorldView-2 images; and the researchers Ph.D. Cléber Gonzales de Oliveira, M.Sc. Emiliano Ferreira Castejon and Ph.D. Thales Sehn Korting for the collaboration on developing this study; the joint work and fruitful discussions with the software development team at PUC-Rio and INPE-DPI is also acknowledged.

\section{REFERENCES}

[1] T. Blaschke, "Object based image analysis for remote sensing". ISPRS Journal of Photogrammetry \& Remote Sensing, No. 65, 2010, pp. 2-16.
[2] B. M. G. Ribeiro, L. M. G. Fonseca, "Evaluation of WorldView-2 Imagery for Urban Land Cover Mapping Using the InterIMAGE System". In: Proceedings of the 4th GEOBIA, 2012, Rio de Janeiro, Brazil, pp. 206-210. Avaiable at: < http://mtc-m18.sid.inpe.br/col/sid.inpe.br/mtc-m18/2012/05.18.16.56/doc/060.pdf>

[3] B. M. G. Ribeiro, L. M. G. Fonseca, H. J. H. Kux, "Mapeamento da Cobertura do Solo Urbano Utilizando Imagens WorldView-II e o Sistema InterIMAGE". Revista Brasileira de Cartografia, Vol. 63, 2011, pp. 51-63.

[4] M.V.A. Carvalho, H.J.H. Kux, T. G. Florenzano, "Urban land cover classification with WorldView-2 images using data mining and Object-Based Image Analysis". In: Proceedings of the 4th GEOBIA, 2012, Rio de Janeiro, Brazil, pp. 431-436.

[5] U.D.V.Souza, H.J.H.Kux, "Contribution of the new WorldView-2 spectral bands for urban mapping in coastal areas: case study São Luís (Maranhão state, Brazil)". In: ISPRS Archives, Vol. XXXIX-B4, 2012, Melbourne, Australia, pp. 171-176.

[6] R. C. Moreira, L. S. Galvão, "Variation in spectral shape of urban materials", Remote Sensing Letters, Vol. 1, No. 3, 2010, pp. 149-158.

[7] T. S. Korting, L. M. G. Fonseca, M. I. S. Escada, F. C. Silva, M. P. S. Silva, "GeoDMA - a novel system for spatial Data mining". In: Data Mining Workshops. IEEE International Conference, 2008.

[8] G. A. O. P. Costa, R. Q. Feitosa, L. M. G. Fonseca, D. A. B. Oliveira, R. S. Ferreira, E. F. Castejon, "Knowledge-based interpretation of remote sensing data with the InterIMAGE system: major characteristics and recent developments". In: Proceedings of the 3rd GEOBIA 2010, Ghent, Belgium.

[9] G. A. O. P. Costa, C. M. D. Pinho, R. Q. Feitosa, H. J. H. Kux, C. M. Almeida, L. M. G. Fonseca, D. Oliveira, "InterIMAGE: an open source platform for automatic image interpretations". In: Proceedings of II Simpósio Brasileiro de Geomática and V Colóquio Brasileiro de Ciências Geodésicas, 2007, Presidente Prudente, Brazil. pp. 735-739.

[10] M. Summerfield. Advanced Qt Programming: Creating Great Software with C++ and Qt 4. Upper Saddle River, N.J.: Addison-Wesley, 2010.

[11] W. Schroeder, K. Martin, B. Lorensen. The Visualization Toolkit: An ObjectOriented Approach To 3D Graphics. New York: Kitware, 2006.

[12] G. Camara, R. M. Souza, B. M. Pedrosa, L. Vinhas, A. M. Monteiro, J. C. Paiva, M. T. Carvalho, M. Gattass, "TerraLib: Technology in support of GIS innovation". In: Workshop Brasileiro de GeoInformática, São Paulo, Brasil, 2000, pp. 126-133.

[13] M. P. S. Silva, G. Câmara, M. I. S. Escada, R. C. M. Souza, "Remote Sensing Image Mining: Detecting Agents of Land Use Change in Tropical Forest Areas". International Journal of Remote Sensing, Vol. 29, No. 16, 2008, pp. 4803-4822.

[14] I. H. Witten, H. Frank. Data mining: practical machine learning tools and techniques with java implementations. San Francisco: Morgan Kaufman, 1999.

[15] InterIMAGE. 2012. InterIMAGE - Interpreting Images Freely. Available at: http://www.lvc.ele.puc-rio.br/projects/interimage/

[16] M. Baatz, A. Schape, "Multiresolution segmentation: an optimization approach for high quality multi-scale image segmentation". In: Proceedings of the XII Angewandte Geographische Informations verarbeitung, Agit Symposium, Salzburg, Áustria, 2000, pp. 12-23.

[17] J. Cohen, "A coefficient of agreement for nominal scales", Educational and Psychological Measurement, No. 20, 1960, pp. 37-46.

[18] G. H. Rosenfield, K. Fitzpatrick-Lins, "A Coefficient of Agreement as a Measure of Thematic Classification Accuracy", Photogrammetric Engineering \& Remote Sensing, Vol. 52, No. 2, 1986, pp. 223-227.

[19] M. Story, R. G. Congalton, "Accuracy assessment: A user's perspective", Photogrammetric Engineering \& Remote Sensing, Vol. 52, No. 3, 1986, pp. 397 399. 